\title{
El Rocoto Relleno de Arequipa, Perú y su Valor Nutricional en la Dieta Alimentaria en el 2021
}

\section{The Stuffed Hot Pepper from Arequipa, Peru and its Nutritional Value in the Food Diet in 2021}

\author{
Carlos Enrique Marchan-Solier \\ Universidad Nacional Autónoma de Huanta, Ayacucho, Perú \\ E-mail: soliersoliercarlos@gmail.com ORCID: https://orcid.org/0000-0003-3912-9015 \\ Ana Fiorela Rico-Taguada \\ Universidad Nacional Autónoma de Huanta, Ayacucho, Perú \\ E-mail: taguadafiorela123@gmail.com ORCID: https://orcid.org/0000-0002-5653-0380 \\ Jhonny de la Cruz-Ocaña \\ Instituto Tecnológico Superior de Villa la Venta, Tabasco, México \\ E-mail: jhonny.c.o@outlook.com ORCID: https://orcid.org/0000-0001-9511-3160 \\ Manuel Alberto Luis Manrique-Nugent \\ Universidad Nacional Autónoma de Huanta, Ayacucho, Perú \\ E-mail: manuelmanriquenu@gmail.com ORCID: $\underline{\text { https://orcid.org/0000-0002-0816-2499 }}$
}

Recepción: 15/06/2021 Aceptación: 30/06/2021 Publicación: 30/07/2021

\section{Resumen}

La presente investigación tuvo como objetivo general determinar el valor nutricional del rocoto relleno de Arequipa, Perú, en el año 2021. La investigación tuvo enfoque cuantitativo, tipo descriptivo, diseño no experimental, nivel correlacional causal, de corte transversal. La población estuvo representada por comensales del recreo campestre Betalleluz en la ciudad de Huanta-Perú; la muestra estuvo conformada por 30 comensales, siendo el muestreo no probabilístico intencional, habiéndose incluido solamente comensales del recreo mencionado que tenga una edad 18 a 65 años y se excluyeron a los clientes que no cumplan con los requisitos determinados en la investigación. La técnica utilizada en la encuesta y los instrumentos para cada variable fueron los cuestionarios. Los resultados indicaron que el rocoto relleno de Arequipa, Perú contiene un valor nutricional alto para la dieta diaria en las personas; como también quedó demostrado que incide significativamente el valor nutricional de los alimentos para la prevención de enfermedades futuras en los seres humanos.

\section{Palabras Clave:}

Rocoto relleno, valor nutricional, Alimentos, enfermedades, dieta. 


\begin{abstract}
The present investigation had as general objective to determine the nutritional value of the hot pepper stuffed from Arequipa, Peru, in the year 2021. The investigation had a quantitative approach, descriptive type, non-experimental design, causal correlational level, cross-sectional. The population was represented by diners from the Betalleluz country recreation in the city of Huanta-Peru; The sample consisted of 30 diners, the sampling being non-probabilistic intentional, having only included diners from the aforementioned recess who were between 20 and 60 years old, and clients who did not meet the requirements determined in the investigation were excluded. The technique used was the survey and the instruments for each variable were the questionnaires. The results indicated that the stuffed hot pepper from Arequipa, Peru contains a high nutritional value for the daily diet in people; as it was also shown that the nutritional value of food has a significant impact on the prevention of future diseases in human beings.
\end{abstract}

\title{
Keywords:
}

Rocoto stuffed, nutritional value, Food, diseases, diet.

\section{Resumo}

A presente investigação teve como objetivo geral determinar o valor nutricional do pimentão recheado de Arequipa, Peru, no ano de 2021. A investigação teve abordagem quantitativa, tipo descritivo, delineamento não experimental, nível causal correlacional, transversal. A população foi representada por comensais da recreação rural Betalleluz da cidade de Huanta-Peru; A amostra foi constituída por 30 comensais, sendo a amostragem não probabilística intencional, tendo-se incluído apenas os clientes do recesso referido com idades compreendidas entre os 18 e 65 anos, sendo excluídos os clientes que não cumpriam os requisitos determinados na investigação. A técnica utilizada na pesquisa e os instrumentos para cada variável foram questionários. Os resultados indicaram que a pimenta recheada de Arequipa, Peru, contém um alto valor nutricional para a dieta diária das pessoas; pois também foi demonstrado que o valor nutricional dos alimentos tem um impacto significativo na prevenção de futuras doenças em humanos.

\section{Palavras-chave:}

Pimenta recheada, valor nutricional, Alimentos, doenças, dieta alimentar.

\section{Introducción}

El ají rocoto (C. pubescens) es una de las especies domesticadas del género Capsicum en Suramérica. Actualmente, su oferta y demanda están en aumento tanto en el mercado 
nacional como global. El objetivo del estudio fue determinar el momento óptimo de extracción de las semillas de C. pubescens en función de su calidad física y fisiológica. Las mediciones de calidad incluyeron el peso de semillas, número de semillas por fruto, emergencia total, taza de emergencia, tiempo medio de emergencia, emergencia media diaria, porcentaje de sobrevivencia, valor pico y valor de emergencia (García-Ruiz et. al, 2018).

La presente investigación plantea como problema fundamental, la siguiente pregunta; ¿Cuál es el valor nutricional del Rocoto relleno arequipeño, en la dieta alimentaria de los comensales en el recreo campestre Betalleluz, en la ciudad de Huanta-Perú?; en este contexto, se plantea el objetivo general, determinar el valor nutricional del Rocoto relleno arequipeño, en la dieta alimentaria de los comensales en el recreo campestre Betalleluz, en la ciudad de Huanta-Perú.

\section{El rocoto relleno de Arequipa y su valor nutricional}

En Capsicum pubescens o coloquialmente denominado el rocoto es una verdura que es cultivado en una gran cantidad en los países de Perú, Chile, Ecuador, Bolivia, México y Argentina. En Latinoamérica se observa que es utilizado el rocoto dentro de la gastronomía nacional y local de los países del continente. (Espinosa et. Al, 2010) afirma que:

“El chile manzano (Capsicum pubescens Ruíz y Pavón) es originario de América del sur, de los Andes, y en México se le cultiva particularmente en las zonas altas, se le encuentra de 1,700 a 2,400. En los últimos diez años se ha observado un incremento en la demanda de este producto, lo cual ha motivado el desarrollo de sistemas de producción intensivo en invernadero para incrementar la cantidad y calidad del fruto." (p.116)

En la gastronomía local de la región de Arequipa, se puede observar que en los potajes que ofrecen al público en general se utiliza con mucha frecuencia los ajíes y rocotos para la elaboración de los platillos tradicionales. (Fuentes, 2015), afirma que "Las variedades principales de ají son el colorado y amarillo que secos toman el nombre de panca y mirasol. En las recetas tradicionales se usan soasados, horneados o hervidos y luego se 
retiran de la cocción". (p.7). En la gastronomía tradicional arequipeña con los rocotos y ajíes se preparan aderezos que se molían en el batán tradicional.

La forma del Capsicum chinense es parecido al del ají amarillo, pero es de color rojo. En el Perú el ají panca se comercializa en su gran porcentaje en polvo como sazonador de los platillos emblemáticos del país. (Baldeón-Apaestegui y Hernández-Gorritti, 2017): “El ají panca posee un sabor picante, debido a la presencia de un grupo de compuestos orgánicos denominados capsaici-noides, de los cuales la capsaicina y la deshidrocapsaicina se encuentran en mayor concentración." (p.224).

Consumir rocoto debidamente no causa gastritis, la capsaicina que contiene actúa protegiendo nuestra mucosa gástrica, los estudios revelan que este compuesto promueve la acumulación de lípidos y bicarbonato formando así una barrera protectora para la mucosa del estómago.(Castañeda \& Salazar, 2014) afirma que:

“La capsaicina (trans-8-metil-N-vanillil-6-nonenamida), es un derivado del ácido homovalínico y representa el componente picante de las especies del género Capsicum .Además, la capsaicina es el constituyente que más se ha estudiado y que ha demostrado tener propiedades analgésicas, antiinflamatorias, antioxidantes, inmunosupresivas y antitumorales" (p.322)

\subsection{La importancia de la correcta dieta alimentaria en la salud}

En el recreo Betalleluz la presente investigación se enfocó en estudiar la dieta alimentaria de los comensales que frecuentan constantemente el lugar mencionado. (Calleja et. al, 2011), afirma que "La obesidad es una enfermedad crónica, compleja y multifactorial que se establece por un desequilibrio entre la ingesta y el gasto energético" (p. 561). En los últimos 2 años se observa que los clientes que frecuentan el recreo Betalleluz tienen sobrepeso y son de una edad promedio 20 a 60 años en gran proporción.

La obesidad, el colesterol, los triglicéridos entre otras enfermedades son los que atacan a los comensales que tienen una edad de 40 a 60 años. (Morales et. al, 2016), afirma que: "La alimentación juega un papel fundamental en el desarrollo de enfermedades crónico degenerativas, cuando la alimentación no es la adecuada se presentan factores de 
riesgo metabólicos constituidos por presión arterial elevada, glucosa elevada, colesterol elevado, sobrepeso y obesidad" (p.22).

En la población de la ciudad de Huanta se cuenta con un problema endémico que es el sobrepeso, la anemia, la hepatitis B, la obesidad entre otros que disminuye la esperanza de vida . (Cabezas et. Al, 2018) afirma que:

La infección por el virus de la hepatitis B (HBV) constituye la décima causa de muerte en el mundo debido a su forma de presentación, provocando casi 600 mil muertes al año. Las complicaciones graves como insuficiencia hepática y hepatocarcinoma se presentan en el $15 \%$ a $25 \%$ del total de pacientes con infección crónica por HBV. A pesar de que existen programas de vacunación, la infección por HBV está presente en toda Latinoamérica. En Perú, las personas no vacunadas tienen la mayor mortalidad por HBV que en todo el continente americano.

En un estudio realizado a 39 familias en la ciudad de Huanta de 15 casos confirmados de infección crónica de HBV se observó que un gran porcentaje no recibía atención médica adecuada (Cabezas et. Al, 2018).

\section{Tabla 1}

Frecuencia de infección por el virus de la hepatitis B en contactos familiares de portadores crónicos según características sociodemográficas.

\begin{tabular}{ccc}
\hline Característica & $\begin{array}{c}\text { Sin infección por HBV } \\
\mathbf{n}(\%)\end{array}$ & $\begin{array}{c}\text { Con infecciónpor HBV } \\
\mathbf{n}(\%)\end{array}$ \\
\hline Edad* & $23,7(18,7)$ & $45(9,8)$ \\
Hombres & $15(93,8)$ & $1(6,2)$ \\
Mujeres & $20(87,0)$ & $3(13,0)$ \\
Vive en zona & $23(88,5)$ & $3(11,5)$ \\
endémica & & \\
Personal de & $3(100,0)$ & $0(0,0)$ \\
salud & $18(94,7)$ & $1(5,3)$ \\
Soltero & $26(89,7)$ & $3(10,3)$ \\
Analfabeto & $35(89,7)$ & $4(10,3)$ \\
\hline Total &
\end{tabular}


Nota: Tabla del estudio de 39 familias en la ciudad de Huanta(Cabezas et. Al,

2018).

En la provincia de Huanta la enfermedad de la hepatitis B es uno de los problemas de salud que no se controla en la zona mencionada. Además, el segundo problema de salud pública es el sobrepeso y obesidad. La correcta dieta alimentaria ayudaría a disminuir las enfermedades en la población local y prolongar la esperanza de vida de los ciudadanos que viven en Huanta. (Alcalde et. al, 2011), Afirma que "Los niños que nacieron en el país en el periodo 1950-1955, por ejemplo, presentaban una esperanza de vida de 44 años" (p.245).

En la ciudad de Huanta la canasta básica se sustenta en la papa, cebada, el trigo, oca, olluco, el arroz, la quinua, la palta, el plátano, entre otros alimentos. (Chapoñan, 2009) afirma que "En el Perú existen más de tres mil variedades y dentro de este grupo encontramos papas nativas y las llamadas "comunes" y "mejoradas", todas de diferentes colores". (p.315).

\section{Gráfico 1}

Total de defunciones, todas las edades

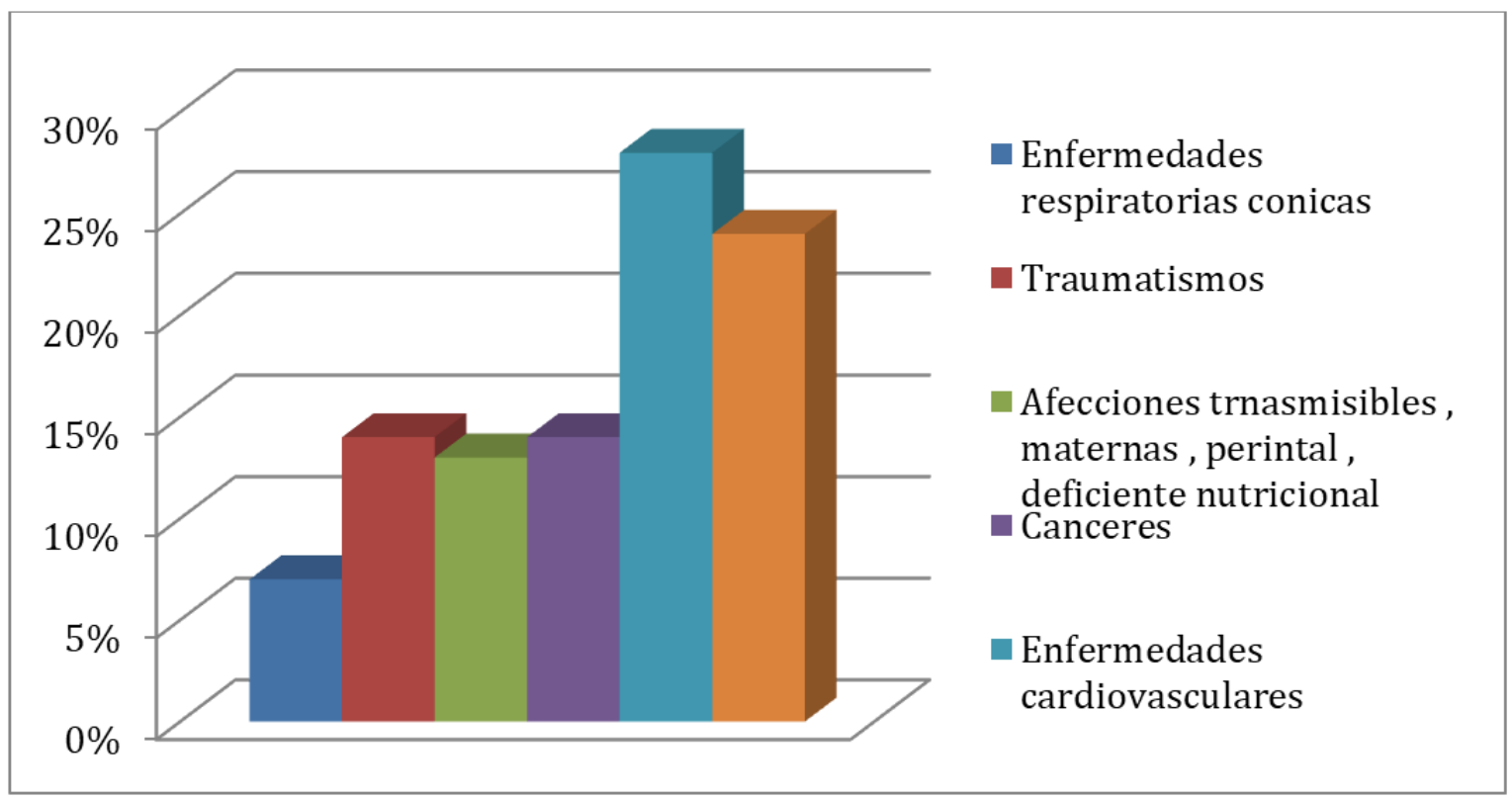

Nota: Organización Mundial de la Salud (OMS, 2014). 
Es recomendable que las personas ingieran alimentos que contengan fibra dietética como cereales integrales, legumbres, vegetales y frutas, con lo que pueden contribuir a reducir el riesgo de desarrollar el síndrome metabólico y enfermedades crónicas como el cáncer, las enfermedades cardiovasculares y la diabetes mellitus tipo 2. (Astovizal, 2010).

\section{Materiales y métodos}

El presente estudio corresponde al enfoque cuantitativo, tipo descriptivo, diseño no experimental, nivel correlacional causal, de corte transversal. Para la obtención de la muestra en el estudio realizado, se utilizó la muestra por conveniencia. Además, la población estuvo conformada por comensales del recreo campestre Betalleluz en la ciudad de Huanta-Perú; la muestra estuvo conformada por 30 comensales, siendo el muestreo no probabilístico intencional, habiéndose incluido solamente comensales del recreo mencionado que tenga una edad 18 a 65 años y se excluyeron a los clientes que no cumplan con los requisitos determinados en la investigación. La técnica utilizada fue la encuesta y los instrumentos utilizados para cada variable fueron cuestionarios. La encuesta tiene 16 preguntas y cada una de ellas cuenta con un promedio de 3 alternativas; la confiabilidad del sondeo de la dieta alimentaria durante la semana de los 30 comensales del recreo campestre Betalleluz arrojo un resultado de 0,936 Y teniendo un grado de confiabilidad muy bueno; la validez se realizó a través de juicio de expertos, quienes refirieron que los instrumentos eran aplicables. La cual permitió establecer la incidencia de una variable independiente sobre otras variables dependiente.

\subsection{Formato de Figuras}

En la tabla 2, se evidencia la distribución de calorías que tienen el plato del rocoto arequipeño en los 15 ingredientes que se necesita para su elaboración; por otro lado, todos los alimentos que son parte de la receta del rocoto relleno fueron pesados en una balanza electrónica en miligramos y convertidos en gramos para realizar los cálculos de cuantas calorías contiene cada comestible mencionado. Asimismo, para el cálculo de las calorías se utilizó la tabla de alimentos de la FDA por tener una aceptación científica mundial de sus 
publicaciones en alimentos y bebidas en el mundo. El resultado total fue que el rocoto arequipeño tiene 517.51865 calorías de acuerdo a la investigación bibliográfica que se realizó con la metodología descrita en el artículo.

\section{Tabla 2}

Cuadro distributivo de las calorías del rocoto relleno arequipeño.

\begin{tabular}{|c|c|c|}
\hline Ingredientes & Gramos & Calorías \\
\hline Rocoto & $170 \mathrm{~g}$ & $52.7 \mathrm{cal}$ \\
\hline Azúcar & $0.015 \mathrm{~g}$ & $0.0583 \mathrm{cal}$ \\
\hline Limón & $0.03 \mathrm{~g}$ & $0.0126 \mathrm{cal}$ \\
\hline Aceite & $14 \mathrm{~g}$ & $130 \mathrm{cal}$ \\
\hline Cebolla & $0.025 \mathrm{~g}$ & $0.008 \mathrm{cal}$ \\
\hline Ajo & $0.015 \mathrm{~g}$ & $0.018 \mathrm{cal}$ \\
\hline Ají panca & $27 \mathrm{~g}$ & $78.84 \mathrm{cal}$ \\
\hline Carne Molida & $40 \mathrm{~g}$ & $96.4 \mathrm{cal}$ \\
\hline Sal & $0.01 \mathrm{~g}$ & $0 \mathrm{cal}$ \\
\hline Orégano seco & $0.005 \mathrm{~g}$ & 0.01325 cal \\
\hline Maní & $5 g$ & $28.35 \mathrm{cal}$ \\
\hline Huevo & $0.06 \mathrm{~g}$ & $0.093 \mathrm{cal}$ \\
\hline Leche & $100 \mathrm{~g}$ & $131 \mathrm{cal}$ \\
\hline Agua & $200 \mathrm{~g}$ & $0 \mathrm{cal}$ \\
\hline Pimienta negra & $0.01 \mathrm{~g}$ & $0.0255 \mathrm{cal}$ \\
\hline
\end{tabular}




\section{Total}

$517.51865 \mathrm{cal}$

Nota: cuadro informativo de las calorías que contiene el plato del rocoto relleno arequipeño (Marchan, 2021).

En la tabla 3, se puede observar el valor nutricional del rocoto relleno arequipeño en los 24 nutrientes que aglomeran el platillo mencionado en su composición alimentaria. En la metodología para la elaboración del valor nutricional del rocoto relleno arequipeño se midió en miligramos los nutrientes con las reglamentaciones de la FDA en el diseño de la tabla №2 en el presente trabajo de investigación.

\section{Tabla 3}

Cuadro distributivo del valor nutricional del rocoto relleno arequipeño

\begin{tabular}{cc}
\hline Nutrientes & Miligramos \\
\hline Grasas saturadas & $41.162 \mathrm{mg}$ \\
Grasas trans & $0.3 \mathrm{mg}$ \\
Colesterol & $33 \mathrm{mg}$ \\
Sodio & $138 \mathrm{mg}$ \\
Proteínas & $47.807 \mathrm{mg}$ \\
Calcio & $375 \mathrm{mg}$ \\
Fosforo & $2213 \mathrm{mg}$ \\
Vitamina A & $240 \mathrm{mg}$ \\
Vitamina D & $15 \mathrm{mg}$ \\
Agua & $18.6 \mathrm{mg}$ \\
\hline
\end{tabular}




\begin{tabular}{|c|c|}
\hline Carbohidratos & $150.791 \mathrm{mg}$ \\
\hline Fibra & $56.34 \mathrm{mg}$ \\
\hline Hierro & $83.1 \mathrm{mg}$ \\
\hline Tiamina & $0.828 \mathrm{mg}$ \\
\hline Riboflavina & $1.919 \mathrm{mg}$ \\
\hline Niacina & $9.7 \mathrm{mg}$ \\
\hline Ácido ascórbico & $76.4 \mathrm{mg}$ \\
\hline Potasio & $34 \mathrm{mg}$ \\
\hline Vitamina B12 & $1 \mathrm{mg}$ \\
\hline Vitamina C & $10 \mathrm{mg}$ \\
\hline Vitamina B6 & $0.2 \mathrm{mg}$ \\
\hline Magnesio & $10 \mathrm{mg}$ \\
\hline Cenizas & $2 \mathrm{mg}$ \\
\hline Zinc & $1 \mathrm{mg}$ \\
\hline
\end{tabular}

Nota: Cuadro informativo de los nutrientes que contiene el plato del rocoto relleno arequipeño (Marchan, 2021).

\section{Resultados}

\subsection{Contrastación de la hipótesis}


Tabla 4

Tabla de comprobación de hipótesis

\begin{tabular}{cc}
\hline Elementos & Promedios \\
\hline Nivel de significancia & 0,814377289 \\
Tamaño de la muestra ( $\mathrm{n})$ & 0.05 \\
Medida muestral & 104 \\
Desviación estándar & 5,38 \\
Estadística de prueba t & 17,13382506 \\
Prueba t stdudent & 5,34 \\
\end{tabular}

\section{Fuente: Elaboración propia.}

Hipótesis general: El valor nutricional del rocoto relleno arequipeño incide significativamente en la dieta alimentaria diaria de los comensales del recreo campestre Betalleluz en el distrito de Huanta-Perú. Hipótesis nula: El valor nutricional del rocoto relleno arequipeño no incide significativamente en la dieta alimentaria diaria de los comensales del recreo campestre Betalleluz en el distrito de Huanta-Perú.

La tabla 4, con los datos obtenidos de la prueba t stdudent se determinó que la hipótesis nula es rechazada en el trabajo de investigación; por lo tanto, aceptando la hipótesis general planteada para la presente investigación científica.

\subsection{Estudio probabilistico del participante $\mathrm{N}^{\circ} 15$}

Igualmente, se tiene los siguientes resultados en la tabla 2, en el cual los datos obtenidos nos muestran que el rocoto relleno arequipeño tiene una composición de 24 nutrientes afirmando que el platillo mencionado tiene un alto componente alimentario en la dieta diaria de los comensales del recreo campestre Betalleluz. Asimismo, en el caso de 
estudio probabilístico del participante №15 se observó que las calorías del rocoto relleno arequipeño están dentro de su distribución alimentaria en el almuerzo confirmando que no causa ningún desequilibrio en su dieta diaria. El índice de masa corporal en el participante №15 está dentro de los parámetros establecidos lo cual afirma que no tienen sobrepeso y pueden incluir el rocoto relleno arequipeño dentro de su dieta alimentaria diaria.

\section{Tabla 5}

Cuadro distributivo las calorías que puede consumir el participante №15 en su dieta alimentaria.

\begin{tabular}{cc}
\hline Datos & Respuestas \\
\hline Edad & 15 \\
Genero & 20 \\
Actividad física & Masculino \\
Peso & Activa \\
\end{tabular}

Nota: cuadro informativo de las calorías que el participante № 15 puede consumir durante el día (Marchan, 2021).

En la tabla № 5, se puede observar los datos generales del participante número 15 que fue seleccionado aleatoriamente realizar el estudio de las calorías diarias que puede consumir en su dieta alimentaria.

\subsubsection{Cálculo de las calorías diarias del participante №15:}

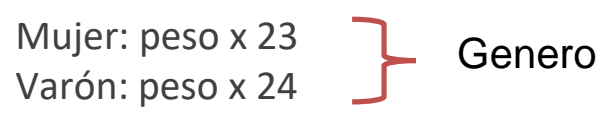




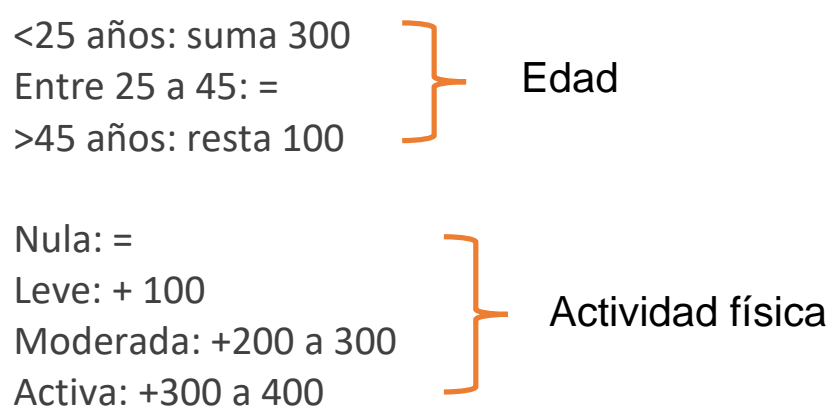

\section{Cálculo de calorías}

Formula:

$$
\begin{aligned}
& \text { Peso } \times \text { Genero }+ \text { Edad }+ \text { Actividad física=Resultado } \\
& 64 \times 24+300+300=2136 \mathrm{kcal} \\
& 2000-2136=136 \mathrm{Kcal}
\end{aligned}
$$

Respuesta.- El participante №15 puede ingerir 2136 kilocalorías de acuerdo al producto de la fórmula planteada en la investigación. La FDA recomienda una ingesta de 2000 kilocalorías lo cual al restar sale como resultado $136 \mathrm{Kcal}$ como excedente de lo recomendado. Asimismo, el excedente mencionado es causa de la intensa actividad física que realiza el participante №15 lo cual no perjudica en su salud de ningún modo.

\subsubsection{Distribución de la dieta alimentaria del participante №15: La distribución alimentaria} se realizara en 3 tiempos de acuerdo a lo recomendado por la FDA en la dieta diaria de una persona promedio. En la investigación el participante №15 puede ingerir 2136 kilocalorías que se distribuyen en el desayuno, almuerzo, cena y extra.

\section{Tabla 6}

Cuadro distributivo de la dieta alimentaria del participante

№15 que debe consumir durante el día en 3 tiempos.

\begin{tabular}{ccc}
\hline Comidas del día & Porcentaje & Kilocalorías \\
\hline Desayuno & $35 \%$ & $747.6 \mathrm{kcal}$ \\
Almuerzo & $30 \%$ & $640.8 \mathrm{kcal}$ \\
\hline
\end{tabular}




\begin{tabular}{ccc}
\hline Cena & $20 \%$ & $427.2 \mathrm{kcal}$ \\
Extra & $15 \%$ & $320.4 \mathrm{kcal}$ \\
\hline Total & & $2136 \mathrm{kcal}$ \\
\hline
\end{tabular}

Nota: Cuadro informativo de la dieta alimentaria que debe consumir el participante №15 en tres tiempos durante el día (Marchan, 2021).

\subsubsection{El índice de masa corporal del participante №15:}

\section{Formula:}

$$
\begin{gathered}
\text { IMC=Peso / altura al cuadrado } \\
\text { IMC=64 kg / (1.69 X1.69) } \\
\text { IMC }=22.408=22.41 \mathrm{imc}
\end{gathered}
$$

Respuesta.- El participante №15 tiene un índice de masa corporal de 22.41 de acuerdo al producto de la fórmula planteada en la investigación. Además, el individuo mencionado tiene $22.41 \mathrm{imc}$ lo cual está considerado como un índice de masa corporal ideal. En conclusión el participante №15 no tiene problemas de sobrepeso y tiene una dieta equilibrada lo cual ayuda a mejorar su salud a largo plazo.

\section{Conclusiones}

De acuerdo a la investigación queda demostrada que el valor nutricional del rocoto relleno arequipeño incide significativamente en la dieta alimentaria diaria de los comensales del recreo campestre Betalleluz en el distrito de Huanta-Perú; lo que implica que la población encuestada opte por el platillo mencionado por su alto nivel nutricional y por tener una dieta alimentaria optima la cual ayuda no tener problemas de salud que reduzcan sus años de vida. 


\section{Referencias}

Espinosa-Torres, L. E., Pérez-Grajales, M. A. R. I. O., Martínez-Damián, M. T., Castro-Brindis, R., \& Barrios-Puente, G. (2010). Efecto de empaques y temperaturas en el almacenamiento de chile manzano (Capsicum pubescens Ruíz y Pavón). Revista Chapingo. Serie horticultura, 16(2), 115-121.

Alcalde-Rabanal, J. E., Lazo-González, O., \& Nigenda, G. (2011). Sistema de salud de Perú. Salud pública de México, 53, s243-s254.

Cabezas, C., Balbuena-Torres, J., Huamaní, L. J. C., Huaman, C. L., Rios-Rojas, J., Idrogo, A. S., \& Anampa-Guzmán, A. (2018). Búsqueda de infección por hepatitis B en familiares de portadores crónicos de la provincia de Huanta, Ayacucho-Perú. Revista Peruana de Medicina Experimental y Salud Pública, 35, 647-651.

Calleja Fernández, A., Muñoz Weigand, C., Ballesteros Pomar, M., Vidal Casariego, A., López Gómez, J. J., Cano Rodríguez, I., ... \& García Fernández, M. (2011). Modificación de los hábitos alimentarios del almuerzo en una población escolar. Nutrición Hospitalaria, 26(3), 560-565.

Chapoñan, M. (2009). ¿La papa engorda? Revista especializada de Nutrición (ReNut), 3(7), 315-317.

Fuentes, M. del C., (2015). LA PICANTERÍA AREQUIPEÑA: PATRIMONIO CULTURAL DE LA NACIÓN.

García-Ruiz, Roger Fabián, Castañeda-Garzón, Sandra Liliana, \& Valdéz-Hernández, Edna Fabiola. (2018). Quality of rocoto pepper (Capsicumpubescens Ruiz \& Pav.) seeds in relation to extraction timing. Acta Agronómica, 67(2), 246-251. https://doi.org/10.15446/acag.v67n2.59057

Morales, M. V., Godoy, S. M., López, M. A. C., \& Alonso, L. G. E. (2016). Enfoque integral de la importancia de la dieta en las condiciones actuales de salud de la población 
mexicana/comprehensive approach of diet importance on health status of the mexican population. BIOtecnia, 18(1), 22-31.

Baldeón-Apaestegui, S.,Hernández-Gorriti, W. (2017). Identificación de la capsaicina y la deshidrocapsaicina en el extracto de oleorresina obtenido a partir de aji panca. Instituto de Investigación Científica, Universidad de Lima. Recuperado de: https://revistas.ulima.edu.pe/index.php/Ingenieria_industrial/article/download/18 $03 / 1818 /$

Astovizal, M. B. (2010). Alimentación adecuada para mejorar la salud y evitar. Revista Cubana de Medicina General Integral, 2.

Castañeda Castañeda, B., \& Salazar Granara, A. A. (2014). Estudio fitoquímico, toxicidad aguda y efectos antiulceroso y antitumoral de los extractos acuoso, etanólico y metanólico de Capsicum pubescens, «Rocoto». 